\title{
FO(ID) as an Extension of DL with Rules
}

\author{
Joost Vennekens ${ }^{\star}$ and Marc Denecker \\ Dept. of Computer Science, K.U. Leuven, Belgium \\ \{joost. vennekens, marc. denecker\}@cs . kuleuven . be
}

\begin{abstract}
There are many interesting Knowledge Representation questions surrounding rule languages for the Semantic Web. The most basic one is of course: which kind of rules should be used and how do they integrate with existing Description Logics? Similar questions have already been addressed in the field of Logic Programming, where one particular answer has been provided by the language of FO(ID). FO(ID) is an extension of first-order logic with a rule-based representation for inductive definitions. By offering a general integration of first-order logic and Logic Programs, it also induces a particular way of extending Description Logics with rules. The goal of this paper is to investigate this integration and discover whether there are interesting extensions of DL with rules that can be arrived at by imposing appropriate restrictions on the highly expressive $\mathrm{FO}(\mathrm{ID})$.
\end{abstract}

\section{Introduction}

Over the past decades, Description Logics (DL) have emerged as an important Knowledge Representation (KR) technology. More recently, they have also had a significant impact on industry, most notably with the adoption of OWL as a W3C standard. In current research, we find a trend to investigate extensions of OWL with rules (e.g. [11]), and, in fact, the hierarchical Semantic Web architecture already prescribes a rule layer on top of the ontology layer formed by OWL. There are a number of interesting KR questions surrounding this topic:

- Which kind of rules are to be used? There are numerous kinds of rules known in the literature (inference rules, rewrite rules, ...), with subtle differences between them.

- What precisely do this kind of rules mean? It should be possible to explain exactly the information content of such a rule and, obviously, this explanation should be consistent with the formal semantics of the rules.

- How do the rules complement $D L$ ? We should be able to clearly indicate how the rules extend the class of knowledge that can be represented by the logic.

In this paper, we will present an answer to these questions based on the language of $\mathrm{FO}(\mathrm{ID})$, a general integration of classical first-order logic (FO) and Logic Programming (LP). Conceptually, FO(ID) is an extension of FO with

\footnotetext{
* Joost Vennekens is a postdoctoral researcher of the FWO.
} 
inductive definitions. To explain, let us consider the following example of an inductive definition, taken from the wikipedia page on the topic1:

The prime numbers can be defined as consisting of:

- 2, the smallest prime;

- each positive integer which is not evenly divisible by any of the primes smaller than itself.

FO(ID) offers a formal syntax for representing such an inductive definition as a set of definitional rules:

$$
\left\{\begin{aligned}
\operatorname{Prime}(2) & \leftarrow \\
\forall x \operatorname{Prime}(x) & \leftarrow x>0 \wedge \neg \exists y y<x \wedge \operatorname{Prime}(y) \wedge \operatorname{Divisible}(x, y)
\end{aligned}\right\}
$$

In natural language, an inductive definition consists of a set of cases in which the defined relation, Prime in this case, holds. Each of these cases corresponds to a definitional rule in the formal syntax of $\mathrm{FO}(\mathrm{ID})$. We remark that the ' $\leftarrow$ 'symbol in the above expression therefore conveys more meaning than the normal material implication of FO: it not only states that certain numbers are prime, but also that no number can be prime unless its "primeness" can be constructively derived by applying the two rules. This additional meaning of such a rule is formalized in FO(ID) using the (parameterized) well-founded model construction from Logic Programming. It was argued in [5] that this formal construction correctly captures the common-sense meaning of such a definition, as it is understood throughout mathematics.

The "job" of an inductive definition is, obviously, to define certain relation(s) (e.g., Prime/1) in terms of some other relation(s) (e.g., $</ 2$ and Divisible/2). In this sense, it has the same semantic status as an FO formula: it expresses a relation between a number of predicates, which may or may not be satisfied by a given interpretation for these predicates. Based on this observation, FO(ID) now integrates inductive definitions into FO: a formula of $\mathrm{FO}$ (ID) is either a regular FO formula or an inductive definition. In this way, FO is extended with a particular form of rules, namely, definitional rules, whose meaning is that each such rule represent a "case" of a particular inductive definition. This enlarges the expressive power of the logic: it is well-known that inductive definitions, such as that of transitive closure, cannot be expressed in FO.

The integration of FO and LP offered by FO(ID) has a number of properties that are quite appealing from the point-of-view of extending DL with rules:

- As opposed to the many hybrid approaches that exist, FO(ID) offers a strong semantic integration of FO and LP.

- In this integration, both components have a clear knowledge representation "task": the LP component is to be used to define concepts, whereas the FO component can be used to assert additional properties of both the defined concepts and concepts for which no definition is provided.

\footnotetext{
${ }^{1}$ http://wikipedia.org/wiki/Inductive_definition
} 
- FO(ID) is particularly natural from a DL perspective: the ability to properly define concepts is already considered to be an important and characteristic feature of $\mathrm{DL} 2$, and FO(ID) essentially just extends it to definitions that cannot be represented by a normal FO equivalence.

- FO(ID) does not impose any arbitrary restrictions on expressivity: it contains full FO and allows general FO formulas in the bodies of its definitional rules.

These properties suggest that FO(ID) is well-suited to serve as a kind of upperbound, from which we can derive meaningful extensions of DL with rules, by imposing suitable restrictions on it. We believe that, in general, this is better than the opposite approach of gradually extending a small tractable language into a more expressive one, since it allows the trade-off between expressivity and complexity to be made more consciously and informedly, which reduces the risk of creating an ad hoc language, whose boundaries are decided more by coincidence than design. In the rest of this paper, we will try to make these claims more concrete, by defining two fragments of FO(ID), that seem to be interesting DL rule languages.

\section{Preliminaries: FO(ID)}

This section summarizes the definition of $\mathrm{FO}$ (ID) as found in [5]. Syntactically, a definition in $\mathrm{FO}(\mathrm{ID})$ is a set of definitional rules, which are of the form:

$$
\forall \boldsymbol{x} P(\boldsymbol{x}) \leftarrow \varphi(\boldsymbol{x}) .
$$

Here, $\varphi(\boldsymbol{x})$ is an FO formula whose free variables are $\boldsymbol{x}$, and ' $\leftarrow$ ' is a new symbol, the definitional implication, which is to be distinguished from the material implication of FO, which we denote by ' $\Leftarrow$ '. We refer to $P(\boldsymbol{x})$ as the head of the rule $r$, denoted head $(r)$, and to the formula $\varphi$ as its body, denoted body $(r)$.

An FO(ID) formula is any expression that can be formed by combining atoms and definitions, using the standard FO connectives and quantifiers. The meaning of the FO formulas and boolean connectives is standard; we therefore only need to define the semantics of a definition in order to define that of $\mathrm{FO}$ (ID).

Let us first introduce some semantical concepts. An interpretation $\mathcal{S}$ for a vocabulary $\Sigma$ consists of a non-empty domain $D$, a mapping from each function symbol $f / n$ to an $n$-ary function on $D$, and a mapping from each predicate symbol $P / n$ to a relation $R \subseteq D^{n}$. A three-valued interpretation $\nu$ is the same as a two-valued one, except that it maps each predicate symbol $P / n$ to a function $P^{\nu}$ from $D^{n}$ to the set of truth values $\{\mathbf{t}, \mathbf{f}, \mathbf{u}\}$. Such a $\nu$ assigns a truth value to each logical atom $P(\boldsymbol{c})$, namely $P^{\nu}\left(c_{1}^{\nu}, \ldots, c_{n}^{\nu}\right)$. This assignment can be extended to an assignment $\nu(\varphi)$ of a truth value to each formula $\varphi$, using the standard Kleene truth tables for the logical connectives:

\footnotetext{
$\overline{2}$ For instance, the DL handbook [1] has the following to say about DL's 'E'-connective: "This form of definition is much stronger than the ones used in other kinds of representations of knowledge, which typically impose only necessary conditions; the strength of this kind of declaration is usually considered a characteristic feature of DL knowledge bases."
} 


\begin{tabular}{|c||c|c|c|c|c|c|}
\hline$\varphi, \psi$ & $\mathbf{t}, \mathbf{t}$ & $\mathbf{t}, \mathbf{f}$ & $\mathbf{t}, \mathbf{u}$ & $\mathbf{u}, \mathbf{f}$ & $\mathbf{u}, \mathbf{u}$ & $\mathbf{f}, \mathbf{f}$ \\
\hline$\varphi \vee \psi$ & $\mathbf{t}$ & $\mathbf{t}$ & $\mathbf{t}$ & $\mathbf{u}$ & $\mathbf{u}$ & $\mathbf{f}$ \\
\hline
\end{tabular}

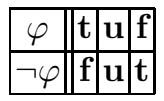

and so on. The three truth values can be partially ordered according to precision: $\mathbf{u} \leq_{p} \mathbf{t}$ and $\mathbf{u} \leq_{p} \mathbf{f}$. This order induces a precision order $\leq_{p}$ on interpretations: $\nu \leq_{p} \nu^{\prime}$ if for each predicate $P / n$ and tuple $\boldsymbol{d} \in D^{n}, P^{\nu}(\boldsymbol{d}) \leq{ }_{p} P^{\nu^{\prime}}(\boldsymbol{d})$

For a predicate $P / n$ and a tuple $\boldsymbol{d} \in D^{n}$, we denote by $\nu[P(\boldsymbol{d}) / \mathbf{v}]$ the threevalued interpretation $\nu^{\prime}$ that coincides with $\nu$ on all symbols apart from $P / n$, and for which $P^{\nu^{\prime}}$ maps $\boldsymbol{d}$ to $\mathbf{v}$ and all other tuples $\boldsymbol{d}^{\prime}$ to $P^{\nu}\left(\boldsymbol{d}^{\prime}\right)$. We also extend this notation to sets $\left\{\left(P_{1}\left(\boldsymbol{d}_{1}\right), \ldots, P_{n}\left(\boldsymbol{d}_{n}\right)\right\}\right.$ of such pairs.

Our goal is to define when a (two-valued) interpretation $\mathcal{S}$ is a model of a definition $\Delta$. We call the predicates that appear in the head of a rule of $\Delta$ its defined predicates and we denote the set of all these by $\operatorname{De} f(\Delta)$; all other symbols are called open and the set of open symbols is written $O p(\Delta)$. The purpose of $\Delta$ is now to define the predicates $\operatorname{Def}(\Delta)$ in terms of the symbols $O p(\Delta)$, i.e., we should assume the interpretation of $O p(\Delta)$ as given and try to construct a corresponding interpretation for $\operatorname{Def}(\Delta)$. Let $O$ be the restriction $\left.\mathcal{S}\right|_{O p(\Delta)}$ of $\mathcal{S}$ to the open symbols. We are now going to construct a sequence of three-valued interpretations $\left(\nu_{\alpha}^{O}\right)_{0 \leq \alpha}$, each of which extends $O$; we will use the limit of this sequence to interpret $\operatorname{Def}(\Delta)$.

- $\nu_{0}^{O}$ assigns $O(P(\boldsymbol{d})) \in\{\mathbf{t}, \mathbf{f}\}$ to $P(\boldsymbol{d})$ if $P \in O p(\Delta)$ and $\mathbf{u}$ if $P \in \operatorname{Def}(\Delta)$;

$-\nu_{i+1}^{O}$ is related to $\nu_{i}^{O}$ in one of two ways:

- Either $\nu_{i+1}^{O}=\nu_{i}^{O}[P(\boldsymbol{d}) / \mathbf{t}]$, such that $\Delta$ contains a rule $\forall \boldsymbol{x} P(\boldsymbol{x}) \leftarrow \varphi(\boldsymbol{x})$ with $\nu_{i}^{O}(\varphi[\boldsymbol{d}])=\mathbf{t}$

- Or $\nu_{i+1}^{O}=\nu_{i}^{O}[U / \mathbf{f}]$, where $U$ is any unfounded set, meaning that it consists of pairs of predicates $P / n$ and tuples $\boldsymbol{d} \in D^{n}$ for which $P^{\nu_{i}^{O}}(\boldsymbol{d})=$ $\mathbf{u}$, and for each rule $\forall \boldsymbol{x} P(\boldsymbol{x}) \leftarrow \varphi(\boldsymbol{x})$, we have that $\nu_{i+1}^{O}(\varphi[\boldsymbol{d}])=\mathbf{f}$.

- For each limit ordinal $\lambda, \nu_{\lambda}^{O}$ is the least upper bound w.r.t. $\leq_{p}$ of all $\nu_{\delta}^{O}$ for which $\delta<\lambda$.

We call such a sequence a well-founded induction of $\Delta$ in $O$. Each such sequence eventually reaches a limit $\nu_{\beta}^{O}$. It was shown in [5] that all sequences reach the same limit. It is now this $\nu_{\beta}^{O}$ that tell us how to interpret the defined predicates. To be more precise, we define that:

$$
\mathcal{S} \models \Delta \text { iff }\left.\mathcal{S}\right|_{\operatorname{Def}(\Delta)}=\left.\nu_{\beta}^{O}\right|_{\operatorname{Def}(\Delta)} \text {, with } O=\left.\mathcal{S}\right|_{O p(\Delta)} .
$$

This tells us when a definition $\Delta$ is satisfied in a structure $\mathcal{S}$. The semantics of full $\mathrm{FO}$ (ID), in which definitions and atoms can be combined using the standard FO connectives, is now simply that which is obtained by adding the usual recursive cases for the connectives (e.g., $\mathcal{S} \models \varphi \wedge \psi$ if $\mathcal{S} \models \varphi$ and $\mathcal{S} \models \psi$, and so). Note that if there is some predicate $P / n$ and $\boldsymbol{d} \in D^{n}$ such that $\nu_{\beta}$ still assigns $\mathbf{u}$ to $P(\boldsymbol{d})$, then the definition has no models extending $O$. Intuitively, this means that, for this particular interpretation of its open symbols, $\Delta$ does not manage to unambiguously define the predicates $\operatorname{Def}(\Delta)$, due to some non well-founded use of negation. 
In the rest of this paper, we will only consider relational vocabularies, that is, there will be no function symbols of arity $>0$.

\section{From FO(ID) to DL(ID)}

As a language that extends full FO, FO(ID) is too expressive to serve as a DL itself. In this section, we discuss the problem of restricting $\mathrm{FO}$ (ID) to a smaller, more DL-like language. In doing this, it is important to be clear about the goals we are trying to achieve. DLs themselves are fragments of FO that are interesting for essentially two reasons:

- They are decidable;

- They are tailored towards a concept-centric modeling style, which they support by means of an intuitive syntax sugar that hides away many of the complexities of FO.

The first of these properties is only relevant for computation, whereas the second concerns knowledge representation. To a certain extent, they are therefore orthogonal concerns.

Most of our attention in this paper will go to the knowledge representation issue, simply because we feel that it comes first. If a logic is to be used at all, for whatever purpose, then theories will have to be written in it. If this is difficult, for instance because the language is too complex or because the meaning of its statements is unclear, then it is unlikely the language will enjoy enduring success, no matter how efficient certain inference task for it might be. Therefore, a primary goal of designing a rule language for DL should be to ensure that it provides a clean and coherent integration of rules into DL, which is easy to understand for those with a working knowledge of DL.

This does not mean that decidability is unimportant, but in the end it is of secondary nature, since also undecidable languages can have interesting uses. Indeed, decidability is only relevant if the goal is to perform deductive inference (i.e., to decide whether, for formulas $\varphi$ and $\psi$, it is the case that $\varphi \models \psi$, in the sense that each model of $\varphi$ is also a model of $\psi$ ) in an unknown or infinite domain. Formally, we know that:

- Given an FO theory $T$ and an FO formula $\varphi$, it is undecidable whether for all structures $S$, it holds that $S \models T$ implies $S \models \varphi$;

- However, given a finite set $D$, an FO theory $T$ and an FO formula $\varphi$, it is decidable (and in co-NP) whether for all structures $S$ such that the domain of $S$ is $D$, it holds that $S \models T$ implies $S \models \varphi$;

When we go from FO to FO(ID), both of these theorems still hold. This is important, because the second situation arizes in numerous interesting applications. For instance, when reasoning in the context of a particular database, we only want to consider the domain $D$ consisting of all the objects in the database. Similarly, if the goal is to query some data from Semantic Web sites, we should 
only consider the domain $D$ of objects about which we are able to retrieve information. A second example is that for many combinatorial problems, the domain is given as part of the specific problem instance that is to be solved; e.g., in graph colouring, $D$ would be the set of nodes, which is fixed once we get a specific graph to colour. Moreover, even when the domain is not known, there might still be approximate algorithms, that are able to derive useful information without guarantees of completeness.

We will therefore now first present a fragment of FO(ID) that allows a DL-like syntactic sugar and offers a DL-like modeling methodology. Only afterwards will we (more briefly) turn to the topic of inference.

\section{A Fragment of $\mathrm{FO}(\mathrm{ID}): \mathcal{A} \mathcal{L C} \mathcal{C}(\mathrm{ID})$}

The goal of this section is to present a fragment of FO(ID), that supports a DLlike modeling style. This will be an extension of the Description Logic $\mathcal{A L C \mathcal { I }}$. More expressive logics, such as $\mathcal{S H O I N}(\mathrm{D})$ which underlies OWL-DL, can be extended in the same way; we restrict to $\mathcal{A L C I}$ merely for simplicity.

Let us first briefly recall $\mathcal{A L C \mathcal { C }}$. We start from a set of unary predicates $\left\{A_{1}, A_{2}, \ldots\right\}$ called (atomic) concepts and a set of binary predicates $\left\{B_{1}, B_{2}, \ldots\right\}$ called (atomic) roles. From the atomic concepts, we build more complex ones using the connectives $\sqcup, \sqcap, \neg, \exists$ and $\forall$; their meaning can be inductively defined by the mapping to FO given in Fig. 1, A role is either an atomic role $B$ or its inverse $B^{-}$, as in Fig. 2. A TBox then consists of a set of inclusions and equivalences as in Fig. 3.

\begin{tabular}{|c|c|l|}
\hline$C$ & $\langle C\rangle(x)$ & \\
\hline$A$ & $A(x)$ & with $A$ an atomic concept \\
$C_{1} \sqcap C_{2}$ & $\left\langle C_{1}\right\rangle(x) \wedge\left\langle C_{2}\right\rangle(x)$ & with $C_{1}, C_{2}$ concepts \\
$C_{1} \sqcup C_{2}$ & $\left\langle C_{1}\right\rangle(x) \vee\left\langle C_{2}\right\rangle(x)$ & with $C_{1}, C_{2}$ concepts \\
$\neg C$ & $\neg\langle C\rangle(x)$ & with $C$ a concept \\
$\forall R . C$ & $\forall y\langle R\rangle(x, y) \Rightarrow\langle C\rangle(y)$ & with $R$ a role and $C$ a concept \\
$\exists R . C$ & $\exists y\langle R\rangle(x, y) \wedge\langle C\rangle(y)$ & with $R$ a role and $C$ a concept \\
\hline
\end{tabular}

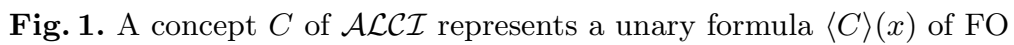

\begin{tabular}{|c|c|l|}
\hline$R$ & $\langle R\rangle(x, y)$ & \\
\hline$B$ & $B(x, y)$ & with $B$ an atomic role \\
$B^{-}$ & $B(y, x)$ & with $B$ an atomic role \\
\hline
\end{tabular}

Fig. 2. A role $R$ of $\mathcal{A L C I}$ represents a binary formula $\langle R\rangle(x, y)$ of FO

\begin{tabular}{|c|c|c|}
\hline$\varphi$ & $\langle\varphi\rangle$ & \multicolumn{1}{|c}{} \\
\hline$C_{1} \sqsubseteq C_{2}$ & $\forall x\left\langle C_{1}\right\rangle(x) \Rightarrow\left\langle C_{2}\right\rangle(x)$ & with $C_{1}, C_{2}$ concepts \\
$C_{1} \equiv C_{2}$ & $\forall x\left\langle C_{1}\right\rangle(x) \Leftrightarrow\left\langle C_{2}\right\rangle(x)$ & with $C_{1}, C_{2}$ concepts \\
\hline
\end{tabular}

Fig. 3. A statement $\varphi$ in a TBox correspond to an FO sentence $\langle\varphi\rangle$ 
We now form the language $\mathcal{A} \mathcal{L C} \mathcal{I}(\mathrm{ID})$ by extending $\mathcal{A} \mathcal{L C} \mathcal{I}$. First, we add the connectives in Fig. 1 for constructing more complex roles: The first three are the obvious analogues of the connectives for concepts, the fourth takes the join of two roles, and the last one takes the Cartesian product of two concepts. Second, and more importantly, we add two new connectives for representing inductive definitions. The first is the symbol ' $\doteq$ ', which is conceptually the same as ' $\equiv$ ', with the difference that it also works for inductive definitions. Formally, it is defined as an abbreviation for an FO(ID) definition containing a single rule, as shown in the first two entries of Fig. 5 .

\begin{tabular}{|c|c|l|}
\hline$R$ & $\langle R\rangle(x, y)$ & \\
\hline$R_{1} \sqcap R_{2}$ & $\left\langle R_{1}\right\rangle(x, y) \wedge\left\langle R_{2}\right\rangle(x, y)$ & with $R_{1}, R_{2}$ roles \\
$R_{1} \sqcup R_{2}$ & $\left\langle R_{1}\right\rangle(x, y) \vee\left\langle R_{2}\right\rangle(x, y)$ & with $R_{1}, R_{2}$ roles \\
$\neg R$ & $\neg\langle R\rangle(x, y)$ & with $R$ a role \\
$R_{1} . R_{2}$ & $\exists z\left\langle R_{1}\right\rangle(x, z) \wedge\left\langle R_{2}\right\rangle(z, y)$ & with $R_{1}, R_{2}$ roles \\
$C_{1} \times C_{2}$ & $C_{1}(x) \wedge C_{2}(y)$ & with $C_{1}, C_{2}$ concepts \\
\hline
\end{tabular}

Fig. 4. Additional ways of constructing roles

For instance, we can define the concept of an uncle as the brother of a parent:

$$
\text { Uncle } \doteq \text { Brother.Parent }
$$

This abbreviates the FO(ID) definition:

$$
\{\forall x, y \operatorname{Uncle}(x, y) \leftarrow \exists z \operatorname{Brother}(x, z) \wedge \operatorname{Parent}(z, y)\}
$$

Such a non-inductive definition (i.e., the role/concept on the left-hand side does not appear in the right-hand side) is equivalent to a regular FO equivalence:

$$
\forall x, y \operatorname{Uncle}(x, y) \Leftrightarrow \exists z \operatorname{Brother}(x, z) \wedge \operatorname{Parent}(z, y) \text {. }
$$

However, ' $\doteq$ ' can also correctly represent inductive definitions. For instance, we can define the role Ancestor as the transitive closure of Parent, by saying that an Ancestor is either a Parent or the Parent of an Ancestor:

\section{Ancestor $\doteq$ Parent $\sqcup$ Parent.Ancestor}

Because of the translation to FO(ID), this too has the correct semantics. Therefore, '' can be used instead of a transitive closure construct such as ${ }^{+}$or the reflexive-transitive closure ${ }^{*}$ of e.g. [12]; it can also replace non-nested uses of the explicit least fixpoint operator $\mu$.

In addition to $\doteq$, we also add the new connective $\leftarrow$ to represent a definitional rule and allow sets of such rules as statements in our language; this is shown in Fig. 5. For instance, extending the notion of an uncle to also include uncles-bymarriage, we could then define this concept by saying that an uncle is 


\begin{tabular}{|c|c|c|}
\hline$\varphi$ & $\langle\varphi\rangle$ & \\
\hline$R_{1} \doteq R_{2}$ & $\left\{\forall x, y\left\langle R_{1}\right\rangle(x, y) \leftarrow\left\langle R_{2}\right\rangle(x, y)\right\}$ & with $R_{1}, R_{2}$ roles \\
$C_{1} \doteq C_{2}$ & $\left\{\forall x\left\langle C_{1}\right\rangle(x) \leftarrow\left\langle C_{2}\right\rangle(x)\right\}$ & with $C_{1}, C_{2}$ roles \\
$\left\{\varphi_{1}, \ldots, \varphi_{n}\right\}$ & $\left\{\left\langle\varphi_{1}\right\rangle, \ldots,\left\langle\varphi_{n}\right\rangle\right\}$ & with $\varphi_{1}, \ldots, \varphi_{n}$ definitional rules (Fig $[6]$ \\
\hline
\end{tabular}

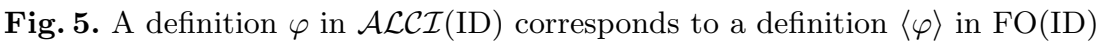

\begin{tabular}{|c|c|c|}
\hline$\varphi$ & $\langle\varphi\rangle$ & \multicolumn{1}{|c}{} \\
\hline$R_{1} \leftarrow R_{2}$ & $\forall x, y\left\langle R_{1}\right\rangle(x, y) \leftarrow\left\langle R_{1}\right\rangle(x, y)$ & with $R_{1}, R_{2}$ roles \\
$C_{1} \leftarrow C_{2}$ & $\forall x\left\langle C_{1}\right\rangle(x) \leftarrow\left\langle C_{2}\right\rangle(x)$ & with $C_{1}, C_{2}$ concepts \\
\hline
\end{tabular}

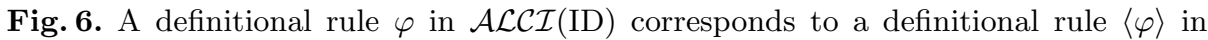
$\mathrm{FO}(\mathrm{ID})$

- Either a brother of a parent;

- Or a husband of an aunt.

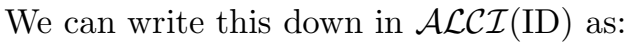

$$
\left\{\begin{array}{l}
\text { Uncle } \leftarrow \text { Brother.Parent } \\
\text { Uncle } \leftarrow \text { Husband.Aunt }
\end{array}\right\}
$$

This abbreviates the following definition in $\mathrm{FO}(\mathrm{ID})$ :

$$
\left\{\begin{array}{l}
\forall x, y \text { Uncle }(x, y) \leftarrow \exists z \operatorname{Brother}(x, z) \wedge \operatorname{Parent}(z, y) \\
\forall x, y \operatorname{Uncle}(x, y) \leftarrow \exists z \operatorname{Husband}(x, z) \wedge \operatorname{Aunt}(z, y)
\end{array}\right\}
$$

In general, if a definition contains multiple rules with the same predicate in the head, these can always be replaced by a single rule whose body is the disjunction of the bodies of the original rules. Therefore, we could rephrase the above definition of $U n c l e$ as

\section{Uncle $\doteq$ Brother.Parent $\sqcup$ Husband.Aunt}

However, this loses the natural case-based structure of the definition, i.e., it makes it more difficult to see that there are actually two separate sufficient conditions, which together also form a necessary condition. Moreover, the rulebased format is also more elaboration tolerant, since rules can more easily be added or removed. For instance, a bank might define the class of persons eligible for a loan as consisting of people with a large income, people who own a house and people with a good credit history; each time the bank now tightens or relaxes its policy, certain rules would have to be removed or added to this definition.

The rule-based representation is also more general than ' $\doteq$ ', since it allows definitions by simultaneous induction as well. For instance, given a two-player game whose move tree is described by the role Successor, we can define the nodes in which I move and the nodes in which my opponent moves by the following simultaneous induction (assuming I start): 


$$
\left\{\begin{array}{l}
\text { HisMove } \leftarrow \exists \text { Successor.MyMove } \\
\text { MyMove } \leftarrow \exists \text { Successor.HisMove } \\
\text { MyMove } \leftarrow \forall \text { Successor. } \perp
\end{array}\right\}
$$

Here, $\perp$ denotes the empty concept.

\section{An Example}

In this section, we will illustrate the language $\mathcal{A L C \mathcal { I }}$ (ID) by means of a simple game. The player is presented a grid of coloured balls. He makes a move by selecting one of these balls. The effect of this is that the entire colour-group to which the ball belongs disappears; the remaining balls then fall down, yielding the next position of the game, as depicted in Fig. 7. The goal of the game is to remove all balls from the grid in such a way as to score as many points as possible. What we will do here is construct an $\mathcal{A L C \mathcal { I }}$ (ID) theory that describes the effect of a single move of this game.
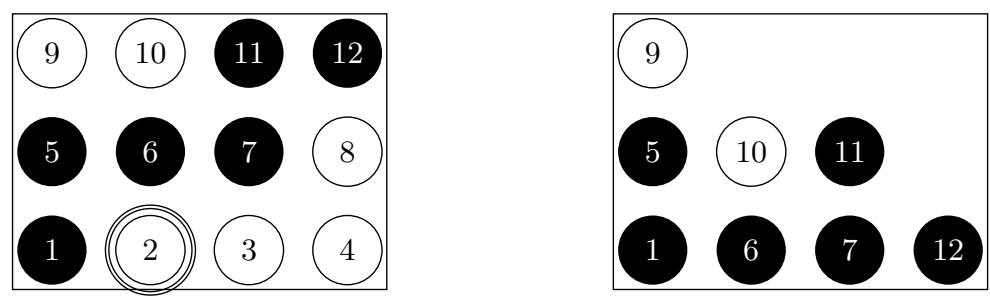

Fig. 7. The result of selecting ball 2

To make things more concrete, let us fix a representation for a state of the game. We represent the grid by roles $U p$ and Left, both with the obvious meaning. The starting grid in Fig. 7, for instance, would correspond to the following interpretations:

$$
\begin{aligned}
& U p=\{(5,1),(9,5),(6,2),(10,6),(7,3),(11,7),(8,4),(12,8)\} ; \\
& \text { Left }=\{(1,2),(2,3),(3,4),(5,6),(6,7), \\
&(7,8),(9,10),(10,11),(11,12)\} .
\end{aligned}
$$

We represent the player's move by a concept Chosen; the move made in Fig. 7 would correspond to Chosen $=\{2\}$. Our goal is now to define roles $U p^{\prime}$ and Left', representing the next state of the game.

We first define some useful auxiliary relations. We begin by defining Above as the transitive closure of $U p$. One ball is above another if it is either directly on top of it, or on top of a ball that is already above it:

$$
\text { Above } \doteq U p \sqcup U p \text {.Above }
$$


A ball is next to another ball if it is either to the left, to the right, underneath, or on top of it:

$$
\text { NextTo } \doteq \text { Left } \sqcup \text { Right } \sqcup U p \sqcup \text { Down }
$$

Of course, Right and Down are the inverses of, respectively Left and Up:

$$
\text { Right } \doteq \text { Left }^{-} \quad \text { Down } \doteq U p^{-}
$$

We remark that of these four definitions, Above is defined inductively, while the others are not. However, in $\mathcal{A L C}($ ID) this difference hardly matters: all four definitions have the same "look and feel".

The balls that disappear after the move are the chosen ball itself and all balls belonging to the same colour-group:

$$
\text { Disappears } \doteq \text { Chosen } \sqcup \exists \text { InColourGroup.Chosen }
$$

Being in the same colour group means being connected through a sequence of balls of the same colour:

$$
\left\{\begin{array}{l}
\text { InColourGroup } \leftarrow \text { SameColour } \sqcap \text { NextTo } \\
\text { InColourGroup } \leftarrow \text { InColourGroup.InColourGroup }
\end{array}\right\}
$$

Here, we use the $\leftarrow$ connective to separate the base case and the inductive step of this definition; the same could be done with our earlier definition of Above.

Two balls have the same colour if the colour of one is also that of the other:

$$
\text { SameColour } \doteq \text { HasColour.HasColour }^{-}
$$

Having now defined which balls disappear, we define the remaining balls as the complement thereof:

$$
\text { Remains } \doteq \neg \text { Disappears }
$$

We now define the relation Above', i.e., the "above"-relation as it will be in the next state. This will hold for any two remaining balls that were originally above each other:

$$
\text { Above }{ }^{\prime} \doteq \text { Above } \sqcap(\text { Remains } \times \text { Remains })
$$

We can now define the relation $U p^{\prime}$ as the intransitive relation of which Above is the transitive closure:

$$
U p^{\prime} \doteq \text { Above }^{\prime} \sqcap \neg\left(\text { Above }^{\prime} \text {. Above }\right)^{\prime}
$$

We define an auxiliary concept OnGround ${ }^{\prime}$ as consisting of those balls that will form the bottom row in the new situation:

$$
\text { OnGround }^{\prime} \doteq \text { Remains } \sqcap \neg \exists \text { Above }^{-} \text {. Remains }
$$

All that remains now is to define Left'. Let us first define when a ball $x$ is in the column to the left of some other ball $y$. For those $y$ that are on a height not greater than that of $x$, this is the case if $x$ is either directly to the left of $y$, or 
below a ball directly to the left of $y$, or above a ball directly to the left of $y$. To cover that situation that $y$ is heigher than $x$ 's, we also add the recursive case that $y$ is above a ball for which we already know that $x$ is in its left column.

$$
\left\{\begin{array}{l}
\text { InLeftColumn } \leftarrow \text { Left } \\
\text { InLeftColumn } \leftarrow \text { Above.Left } \\
\text { InLeftColumn } \leftarrow \text { Above }{ }^{-} \text {.Left } \\
\text { InLeftColumn } \leftarrow \text { LeftColumn.Above' }
\end{array}\right\}
$$

We now define Left' as consisting of all pairs of balls such that (1) one ball is in the column to the left of the other, and (2) both are on the same height:

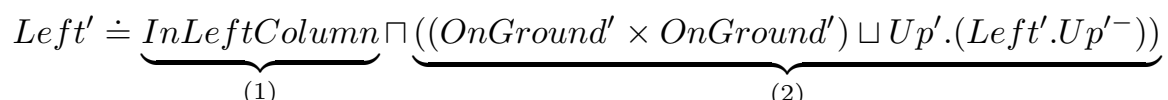

Note that this too is an inductive definition, which proceeds along the rows of the grid: the base case is the bottom row, whereas the inductive case defines that $x$ is to the left of $y$ in the following situation:

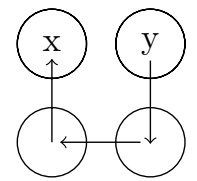

This concludes our representation of the game. As this example shows, $\mathcal{A L C I}(\mathrm{ID})$ can represent many different kinds of definitions (case based definitions, non-inductive definitions, monotone inductive definitions, non-monotone inductive definitions) in a uniform way in, leading to a clean and coherent language.

\section{$6 \quad$ Inference in $\mathcal{A} \mathcal{L C} \mathcal{I}($ ID)}

We lack space for a proof, but it is not hard to show that $\mathcal{A} \mathcal{L C I}$ (ID) is undecidable. However, this does not mean that is cannot be used in practice.

\subsection{Model Expansion}

[13. considered the inference task of model expansion for $\mathrm{FO}(\mathrm{ID})$ : given an interpretation $\mathcal{S}$ for some subset $\Sigma_{0}$ of the alphabet $\Sigma$ of a theory $T$, extend $\mathcal{S}$ with an interpretation for the remaining symbols $\Sigma \backslash \Sigma_{0}$, such that the resulting interpretation is a model of $T$. Here, $\mathcal{S}$ is required to have a finite domain. Note that, in general, a model expansion problem may have many different solutions, because even though $\mathcal{S}$ has to fix the domain of discourse, it does not fix the interpretation of $\Sigma \backslash \Sigma_{0}$ in this domain, but only restrains it to satisfy $T$. This form of inference is decidable for full $\mathrm{FO}$ (ID) and in fact captures the complexity class NP. 
To illustrate the usefulness of this inference task, let us consider again the example of the previous section. Here, we defined the next state of a game $\left(L e f t^{\prime}\right.$ and $\left.U p^{\prime}\right)$ in terms of its old state (Left and $\left.U p\right)$ and a given move (Chosen). We can therefore compute a new state of the game by performing model expansion on the structure $\mathcal{S}$ for the alphabet $\Sigma_{0}=\{$ Left, Up, Chosen $\}$. Because our representation of the game defines all of its predicates except the ones in $\Sigma_{0}$, this computation can actually be done in polynomial times.

The IDP-system 3 implements the task of model generation of FO(ID). A program transforming $\mathcal{A L C \mathcal { I }}(I D)$ syntax to input for this system is available 4 Together, these two programs are able to, for instance, use our formalizaton of Section 5 to compute state transitions for this game.

\subsection{Guarded $\mathcal{A} \mathcal{L C}$ (ID)}

As discussed, there are interesting applications, that only require reasoning in a fixed, finite domain and for which the undecidability of $\mathrm{FO}$ (ID) is therefore not a problem. However, because there are of course also other cases, this section will develop a decidable fragment of $\mathrm{FO}(\mathrm{ID})$.

This fragment is based on the guarded fragment of FO. We recall that an FO formula $\psi$ is guarded if every one of its quantified subformulas is either of the form $\exists \boldsymbol{x} G(\boldsymbol{x}, \boldsymbol{y}) \wedge \varphi(\boldsymbol{x}, \boldsymbol{y})$ or $\forall \boldsymbol{x} G(\boldsymbol{x}, \boldsymbol{y}) \Rightarrow \varphi(\boldsymbol{x}, \boldsymbol{y})$, where $G(\boldsymbol{x}, \boldsymbol{y})$ is an atom, called the guard, such that the free variables $\operatorname{free}(\varphi(\boldsymbol{x}, \boldsymbol{y})) \subseteq \operatorname{free}(G(\boldsymbol{x}, \boldsymbol{y}))$.

We now define a similar guarded fragment of $\mathrm{FO}(\mathrm{ID})$. This fragment allows only theories consisting of precisely one definition and precisely one FO formula.

Definition 1. Let $T$ be an $F O(I D)$ theory, consisting of precisely one definition $\Delta$ and one $F O$ formula $\varphi$. $T$ is guarded if

- $\varphi$ is a guarded formula;

- for each rule $\forall \boldsymbol{x} P(\boldsymbol{x}) \leftarrow \psi$, it holds that $\psi$ is a guarded formula and $\boldsymbol{x} \subseteq$ $\operatorname{free}(\psi)$;

- none of the guards is defined by $\Delta$.

Exploiting a theorem from [8], we can prove the following result.

Theorem 1. The guarded fragment of $F O(I D)$ is decidable. More precisely, deductive reasoning for $F O(I D)$ is $2 \mathrm{EXPTIME-complete.}$

Proof. Omitted for space.

This of course raises the question of how this guarded fragment of $F O(I D)$ relates

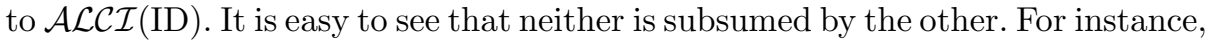
$P \doteq P . P$ translates to

$$
\{\forall x, y P(x, y) \leftarrow \exists z P(x, z) \wedge P(z, y) \cdot\},
$$

\footnotetext{
${ }^{3}$ http://www.cs.kuleuven.be/ $\sim$ dtai/krr/software/idp.html

4 http://www.cs.kuleuven.be/ joost/alc_id.tar.gz
} 
which is not guarded. Conversely, the guarded fragment is not subsumed by $\mathcal{A L C \mathcal { I }}$ (ID) either, since, for instance, the latter only allows unary and binary predicates, while the former allows arbitrary arities.

The two fragments do, however, have a significant intersection. Let us consider a theory $T$ in $\mathcal{A L C I}$ (ID) and examine which additional conditions can ensure that its translation is guarded.

The guarded fragment of $\mathrm{FO}$ (ID) only allows theories to contain a single definition, whereas an $\mathcal{A L C} \mathcal{L}$ (ID) theory can have multiple definitions. In [17, we examined when a set of definitions $\left(\Delta_{i}\right)_{1<i<n}$ can be merged into a single equivalent definition $\cup_{i} \Delta_{i}$. We showed that the following condition is sufficient.

Condition 1. There exists a preorder $\leq$ on the predicates of the theory, such that for all $\Delta_{i}$ and $P \in \operatorname{Def}\left(\Delta_{i}\right)$ :

- if $Q \in \operatorname{Def}\left(\Delta_{i}\right)$ then $P \geq Q$;

- if $Q$ appears in $\Delta_{i}$ but does not belong to Def $\left(\Delta_{i}\right)$, then $P>Q$ (i.e., $P \geq Q$ and $P \not \leq Q)$.

By imposing this condition, we can merge all definitions into a single definition, thus already satisfying that particular requirement of our guarded fragment. Looking at the translation of $\mathcal{A L C \mathcal { I }}$ (ID) into $\mathrm{FO}$ (ID), it is quite clear that the only other cause for falling outside of the guarded fragment is the fact that defined predicates might be used as guards.

Condition 2. For every construct $\exists$ R.C, $\forall$ R.C or R.S that appears in $T$, it must be the case that $R$ is not defined, i.e., $T$ does not contain any formulas of the form $R \doteq \varphi$ or any definitions in which a rule $R \leftarrow \varphi$ appears.

We will say that an $\mathcal{A L C I}$ (ID) theory is guarded if it satisfies both Condition 1 and Condition 2 .

Theorem 2. Let $T$ be a guarded $\mathcal{A L C I}(I D)$ theory and let $T^{\prime}$ be the result of merging all definitions in $\langle T\rangle$ into a single definition. Then $T^{\prime}$ is equivalent to $\langle T\rangle$ itself and belongs the guarded fragment of FO(ID).

We remark that our Condition 2 has a somewhat similar flavour to the decidable fragment of SWRL that is formed by (strongly) safe rules [15]: a SWRL-rule (i.e., Horn clause) is safe if every one of its variables appears in an atom whose predicate is not used anywhere in the TBox (i.e., it may only appear in other rules or in the ABox). This too requires that variables are "guarded" by atoms about which there is not "too much" information present in the theory.

\section{Related Work}

The most widely known DL rule-language is currently that of SWRL [1], which extends OWL with Horn clauses. Since its rules only state sufficient conditions, and cannot express necessary ones, they cannot be used to define concepts. Because definitions are an important feature of DL (see footnote on $\mathrm{p}$ 386), 


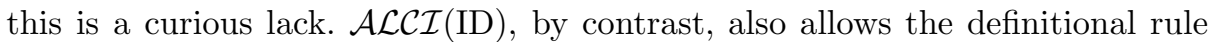
construct $\leftarrow$, in addition to $\sqsupseteq$. Moreover, it also allows more that just Horn clauses, since the body of a rule may contain negation and quantifiers.

In [6], a combination of DL and LP under the well-founded semantics is investigated, while [7] presents an integration of DL and ASP. Unlike FO(ID)'s semantic integration of LP and DL, these two approaches foster a strong separation between the two components: essentially, they allow a logic program to pose queries to a description logic theory, with the latter acting more-or-less as a black box towards the former. In contrast, FO(ID) provides a full semantic integration in which both both logic programs and description logic axioms are first-class citizens.

Description logic programs [9] and $\mathcal{D} \mathcal{L}+\log$ [16] are two strong integrations, which both consider only a quite restricted language, with Grosof et al. focusing on the intersection, rather than the union, of DL and LP, and Rosati only considering Datalog. By contrast, FO(ID) allows full first-order logic in the body of rules, using the machinery of the well-founded semantics to properly handle negation.

[3], 14] and [10] both provide strong semantic integrations for a more general kind of rules, with 10 also defining a guarded fragment that, like ours, can be mapped into guarded fixpoint logic. The main feature that distinguishes our approach from these is of a KR nature. We offer rules that have a clear and intuitive interpretation as definitional rules. On the one hand, this means that it easy to explain the total information content of such a rule: it represents a "case" of an (inductive) definition. On the other hand, we also have clear guidelines as to what they should be used for: the definitional rules should be used to define concepts, and other statements (regular inclusions) can serve to state additional properties, such conditions that are only sufficient. The KR picture for the other approaches is less clear.

\section{Conclusions and Future Work}

In this paper, we have investigated the extension of DL with rules that is induced by $\mathrm{FO}(\mathrm{ID})$. We first argued that this is quite natural for the following reasons:

- There is an appealing match between the intuitive notion of a "case" in an inductive definition and the formal construct of a definitional rule in FO(ID);

- Since non-inductive definitions are already a key feature of DL, it makes sense to exploit this match by adding definitional rules to DL, in order to extend the class of definitions that can be represented.

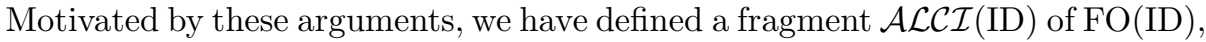
which offers a DL-like syntax for representing (inductive) definitions. For FO(ID), and therefore also for $\mathcal{A L C I}$ (ID), there exist useful inference tasks, such as model expansion, that can be performed efficiently (see Section 6 This is despite the fact that the language as a whole is undecidable for deductive inference. We have defined a decidable guarded fragment of $\mathcal{A L C I}$ (ID). A more comprehensive analysis 
of this and other decidable fragments of $\mathrm{FO}$ (ID) is left for future work. The goal of the current paper is not to present a single DL rule-language that is suitable for all purposes, but rather to point towards $\mathrm{FO}$ (ID) in general as an interesting foundation from which such languages can be derived.

\section{References}

1. Baader, F., Calvanese, D., McGuinness, D., Nardi, D., Patel-Schneider, P. (eds.): The Description Logic Handbook. Theory, Implementation and Applications. Cambridge University Press, Cambridge (2002)

2. Brachman, R.J., Levesque, H.J.: Competence in Knowledge Representation. In: AAAI, pp. 189-192 (1982)

3. de Bruijn, J., Pearce, D., Polleres, A., Valverde, A.: Quantified equilibrium logic and hybrid rules. In: Marchiori, M., Pan, J.Z., Marie, C.d.S. (eds.) RR 2007. LNCS, vol. 4524, pp. 58-72. Springer, Heidelberg (2007)

4. Denecker, M., Ternovska, E.: A logic of non-monotone inductive definitions. Transactions On Computational Logic (TOCL) (2008)

5. Denecker, M., Vennekens, J.: Well-founded semantics and the algebraic theory of non-monotone inductive definitions. In: Baral, C., Brewka, G., Schlipf, J. (eds.) LPNMR 2007. LNCS, vol. 4483, pp. 84-96. Springer, Heidelberg (2007)

6. Drabent, W., Henriksson, J., Maluszynski, J.: HD-rules: a hybrid system interfacing Prolog with DL-reasoners. In: 2nd Int'l. Workshop on Applications of Logic Programming to the Web, Semantic Web and Semantic Web Services (2007)

7. Eiter, T., Lukasiewicz, T., Schindlauer, R., Tompits, H.: Combining answer set programming with description logics for the semantic web. In: KR (2004)

8. Grädel, E., Walukiewicz, I.: Guarded fixed point logic. In: LICS, pp. 45-55 (1999)

9. Grosof, B., Horrocks, I., Volz, R., Decker, S.: Description logic programs: combining logic programs with description logic. In: WWW (2003)

10. Heymans, S., Van Nieuwenborgh, D., Vermeir, D.: Open Answer Set Programming with guarded programs. ACM TOCL 9(4) (2008)

11. Horrocks, I., Patel-Schneider, P.F., Boley, H., Tabet, S., Grosof, B., Dean, M.: SWRL: A semantics web rule language combining OWL and RuleML. W3C Submission (2004), http://www.w3.org/Submission/2004/SUBM-SWRL-20040521/

12. De Giacomo, G., Lenzerini, M.: Tbox and ABox reasoning in expressive description logics. In: KR, pp. 316-327. Morgan Kaufmann, San Francisco (1996)

13. Mitchell, D., Ternovska, E.: A framework for representing and solving NP search problems. In: AAAI, pp. 430-435. AAAI Press/MIT Press (2005)

14. Motik, B., Rosati, R.: A faithful integration of description logics with logic programming. In: IJCAI (2007)

15. Motik, B., Sattler, U., Studer, R.: Query answering for OWL-DL with rules. In: McIlraith, S.A., Plexousakis, D., van Harmelen, F. (eds.) ISWC 2004. LNCS, vol. 3298, pp. 549-563. Springer, Heidelberg (2004)

16. Rosati, R.: DL+log: Tight integration of description logics and disjunctive datalog. In: KR, pp. 68-78 (2006)

17. Vennekens, J., Denecker, M.: An algebraic account of modularity in ID-logic. In: Baral, C., Greco, G., Leone, N., Terracina, G. (eds.) LPNMR 2005. LNCS, vol. 3662, pp. 291-303. Springer, Heidelberg (2005) 